\title{
Biogenic Synthesis of Silver Nanoparticles Using Saraca indica Leaf Extract and Evaluation of Their Antibacterial Activity
}

\author{
Ravi Mani Tripathi ${ }^{\mathrm{a}, \mathrm{b}, \mathrm{c}}$, Deepti Rana ${ }^{\mathrm{c}}$, Archana Shrivastav ${ }^{\mathrm{a},{ }^{*}}$, Rajendra Pal Singh $^{\mathrm{c}}$, Braj Raj Shrivastav ${ }^{\mathrm{d}, \mathrm{e}}$ \\ ${ }^{a}$ Department of Microbiology, College of Life Sciences, Gwalior - 474009 (M.P.), India. \\ ${ }^{\mathrm{b}}$ School of Science in Biotechnology, Jiwaji University, Gwalior - 474011 (M.P.), India \\ ${ }^{c}$ Amity Institute of Nanotechnology, Amity University, Sector- 125, Noida- 201303, India \\ ${ }^{\mathrm{d} D e p a r t m e n t ~ o f ~ S u r g i c a l ~ O n c o l o g y, ~ C a n c e r ~ H o s p i t a l ~ \& ~ R e s e a r c h ~ I n s t i t u t e, ~ G w a l i o r ~-~} 474009$ (M.P.), India. \\ ${ }^{\mathrm{e}}$ Department of Surgery, G. R. Medical College, Palace Road, Gwalior - 474009 (M.P.), India \\ * Corresponding author: archana_61@yahoo.co.in \\ Tel/fax.: +91 7512336502
}

\begin{abstract}
The present study reports an unprecedented biogenic method for the synthesis of silver nanoparticles (AgNPs) using leaf extract of Saraca indica and characterized their antibacterial activity. We have also focused on the biosynthesis mechanism of AgNPs. Plant leaf extract has water soluble organic materials which help in the reduction of silver ions and stabilization of AgNPs. Aqueous solution of silver nitrate was treated with leaf extract of Saraca indica for the formation of AgNPs. The surface plasmon resonance was occurred at $412 \mathrm{~nm}$. The size distribution profile of synthesized AgNPs was analysed by Dynamic Light Scattering (DLS). Transmission Electron Microscopy (TEM) has been done for the measurement of particle size and their morphology. The role of phytochemicals in the reduction of silver ions and defining the framework in which AgNPs are covered and provide steadiness can be determined through Fourier Transform Infrared Spectroscopy (FTIR). TEM micrograph reveals that the size of AgNPs was obtained in the range of 13-50 nm with spherical morphology. X-ray Diffraction (XRD) pattern of the AgNPs exhibited $2 \theta$ values corresponding to the silver nanocrystals. Furthermore, the antibacterial activity of synthesized AgNPs against E. coli DH5 $\alpha$ was investigated by growth curve and inhibition zone analysis. It was observed that the $20 \mu \mathrm{g} / \mathrm{ml}$ concentration of biogenic AgNPs recorded as minimal inhibitory concentration (MIC) against $E$. coli DH5 $\alpha$.
\end{abstract}

Keywords: Silver nanoparticles, Saraca indica, E. coli DH5 $\alpha$, Antibacterial activity, Well diffusion method

Citation: R.M. Tripathi et al. Biogenic Synthesis of Silver Nanoparticles Using Saraca indica Leaf Extract and Evaluation of Their Antibacterial Activity. Nano Biomed. Eng. 2013, 5(1), 50-56. DOI: $10.5101 /$ nbe.v5i1.p50-56.

\section{Introduction}

The synthesis of metal and semiconductor nanoparticles is an expanding research area, due to their potential applications for the development of novel technologies [1]. Basically, nanotechnology deals with the synthesis of nanoparticles of different shapes, sizes and their potential applications [2]. Nanoparticles have a wide range of applications in the field of environmental pollution control, drug delivery, and material chemistry [3]. However, the augmentation of a suitable process or mechanism of nanomaterial synthesis as well as full control of size and dispersity is still a matter of great confront to current nanotechnology [4-8]. Synthesis of metal nanoparticles has an advantage of having the large surface area for their antimicrobial activity and other applications [1]. For thousands of years silver has been used as a healing and antimicrobial agent throughout the world [9]. Some researchers have shown silver's

magisterial performance in fighting against microbes [10]. Ionic silver can be used in wide applications from antimicrobial material to electronic sensors [11].

There are several methods existing for the synthesis of nanoparticles such as using chemical reducing agents with enhancers, bacteria, fungus, many other microbes and plant parts extracts. Among the various known synthesis methods, plant-mediated nanoparticles synthesis is preferred as it is cost-effective, environment friendly and safe for human therapeutic use $[12,13]$. The chemical method for the synthesis of nanoparticles has several occupational exposure hazards like carcinogenicity, genotoxicity, cytotoxicity and general toxicity [14]. In the use of microbes, the problem for bio reduction is to maintain the aseptic conditions, which not only requires technical labour but also boosts the production cost. It has been reported that medicinally valuable angiosperms 
have the greatest potential for synthesis of metallic nanoparticles with respect to quality and quantity [1215]. Many plant extract have the potential ability to synthesize AgNPs extra-cellularly including benghalensis [1], Syzygium cumini [2], Pelargonium graveolens [16], Capsicum annuum [17], Azadirachta indica [18], Allium cepa [19], Ficus coffee and tea (Camellia sinensis) [20], Mentha piperita [21] and Brassica juncea [22].

It was found experimentally that the polyol components and water soluble hydro cyclic compounds present in leaf were mainly responsible for reduction of silver ions [23]. Biosynthesized silver nanoparticles (AgNPs) are used in label free colorimetric assay [24], water-filtering apparatus [25], catalysis [26], anti-viral and anti-HIV studies [27]. Many researchers have tried to synthesize AgNPs of variable size, shape and dispersity by varying metal ion or leaf broth concentration, $\mathrm{pH}$, and temperature $[12-15,18]$. It has been hypothesized that several factors together determine the synthesis of nanoparticles such as plant source, organic compounds in the crude plant extract and even the pigments, alkaloids, polyphenols and proteins etc. in plant extract $[13,18]$.

In the present study, we have described the mechanistic approach of biosynthesis of AgNPs using Saraca indica leaf extract. The antibacterial activity of synthesized AgNPs against $E$. coli $\mathrm{DH} 5 \alpha$ was investigated by growth curve and well diffusion method.

\section{Experimental}

\subsection{Chemicals \& bacteria}

Saraca indica plant leaves were collected from the garden of Amity University, Noida, India, for the synthesis of AgNPs. The silver nitrate $\left(\mathrm{AgNO}_{3}\right)$ was purchased from Merck limited, India. Lyophilized culture of $E$. coli DH5 $\alpha$ was obtained from Department of Microbiology, College of Life Sciences, Gwalior, India. Luria Bertani media was supplied by Hi-Media Laboratories, Bombay, India.

\subsection{Synthesis of AgNPs}

Leaf extract was prepared by taking $25 \mathrm{~g}$ of thoroughly washed fresh leaves of Saraca indica. These leaves were crushed by a piston and mortar till a paste is formed. Then pour the paste to a $500 \mathrm{ml}$ flask and add $100 \mathrm{ml}$ of deionised water to it. Then this solution is allowed to attain a temperature of $60{ }^{\circ} \mathrm{C}$ for $15-25$ minutes. Subsequently, the solution is filtered by using a filter paper and the filtrate thus obtained is the required leaf extract solution. For the synthesis of AgNPs, $1 \mathrm{mM}$ $\mathrm{AgNO}_{3}$ solution was prepared in an Erlenmeyer flask. This solution was sustained at a temperature of 60 $80^{\circ} \mathrm{C}$ for 10 minutes. Subsequently, $5 \mathrm{ml}$ of plant extract solution was added drop by drop along with constant stirring. After some time, a colour change is observed this indicates the initiation of nanoparticles synthesis. The obtained solution is stable for around 5-6 weeks.

\subsection{Instrumentation}

\subsubsection{UV-Vis spectroscopy}

UV-Vis absorption spectroscopy is the branch of spectroscopy where the absorption of light by molecules that are in a gas or vapor state or dissolved molecules/ ions can be measured. It investigates the absorption between the wavelength limits $190 \mathrm{~nm}$ and $1100 \mathrm{~nm}$, where the absorption of the electromagnetic radiation is caused by the excitation of bonding and non-bonding electrons of the ions or molecules. The measured sample's absorption spectrum is continuous due to the fact that the different vibration and rotation states of the molecules make the absorption band wider. A UV-1601 pc Shimadzu spectrophotometer is used to study the absorption spectrum of the AgNPs synthesized by Saraca indica leaf extract. The reaction mixture is analyzed in the wavelength range from 200-800 $\mathrm{nm}$.

\subsubsection{Dynamic light scattering (DLS)}

DLS (also known as Quasi-Elastic Light Scattering) is a technique which can be used to determine the size distribution profile of particles in suspension or polymers in solution. This measurement depends on the size of the particle nucleus, the size of surface structures, particle concentration, and the type of ions in the medium. Since DLS essentially measures fluctuations in scattered light intensity due to diffusing particles, the diffusion coefficient of the particles can be determined.

\subsubsection{Transmission electron microscopy (TEM)}

TEM uses an electron beam to prepare an image of a sample. High energy electron beam falls upon ultrathin samples allow for image resolutions that are of the order of 1-2 A. The morphology and size of AgNPs were characterized by TEM (Philips CM-10). A thin film of the sample was prepared onto a carbon coated copper grid. A beam of electrons is transmitted through an ultra thin specimen. These electrons interact with the specimen and form an image. The image is magnified and focused onto an imaging device, such as a fluorescent screen, on a layer of photographic film.

\subsubsection{Fourier transform infrared spectroscopy (FTIR)}

In FTIR spectroscopy, IR radiation is passed through a sample. Some of the part is absorbed by the sample and another is passed through it. The resulting spectrum signifies the molecular absorption and transmission which creates the molecular fingerprint of the sample. This fingerprint spectrum with absorption peaks corresponds to the frequencies of vibrations between the bonds of the atoms of sample materials. In addition, the size of the peaks in the spectrum is a direct indication of the amount of material present. A Perkin-Elmer 1750, Norwalk, CT (FTIR) is used to analyse the sample solution of AgNPs along with the phytochemical that are possibly responsible for the reduction and stabilization of the nanoparticles. 


\subsubsection{X-ray diffraction (XRD)}

X-ray diffraction (XRD) analysis of powdered AgNPs in sample was obtained for the determination of the formation of AgNPs by analyzing the peak intensity, position and width. It helps to identify the crystalline material, unit cell dimensions and sample purity. Scherrer's formula is used to determine the mean particle size using full width at half-maximum (FWHM) data provided in the graph.

$$
d=0.9 \lambda / \beta \cos \theta
$$

Where $d$ is the mean diameter of the nanoparticles, $\lambda$ is the wavelength of X-ray radiation source and $\beta$ is angular FWHM of the XRD peak at diffraction angle $\theta$.

\subsection{Evaluation of antibacterial activity of AgNPs}

The antibacterial activity of AgNPs was evaluated against $E$. coli DH5 $\alpha$, a gram-negative bacterium. Consequence of AgNPs on bacterial growth was monitored at a regular interval of $5 \mathrm{~h}$ (up to $25 \mathrm{~h}$ ) by measuring the optical density (OD). Bacterial culture was supplemented with different concentration of AgNPs ranging from $20-45 \mu \mathrm{g} / \mathrm{ml}$. The control culture was treated as in the same fashion but without any exposure of AgNPs. Afterward, the culture was incubated at $37{ }^{\circ} \mathrm{C}$ for around $25 \mathrm{~h}$. The growth of E. coli DH5 $\alpha$ in broth media was indexed by measuring the OD (at wavelength of $600 \mathrm{~nm}$ ) at regular intervals using UV-Vis spectrophotometer.

Further the antibacterial activity of biogenic AgNPs was also assayed by well diffusion method. The zone at which no bacterial colonies can be grown are called inhibition zone. The inhibition zone depends upon the concentration of nanoparticles if we increase the concentration then inhibition zone will also increase. LB agar was prepared and poured into the petri-plates after solidification wells were prepared. Subsequently, broth culture of $E$. coli DH5 $\alpha$ was spread on the surface of media and the AgNPs solution with concentrations ranging from $20-45 \mu \mathrm{g} / \mathrm{ml}$ was poured into the wells and allowed to incubate for $24 \mathrm{~h}$ at $37^{\circ} \mathrm{C}$.

\section{Results and discussion}

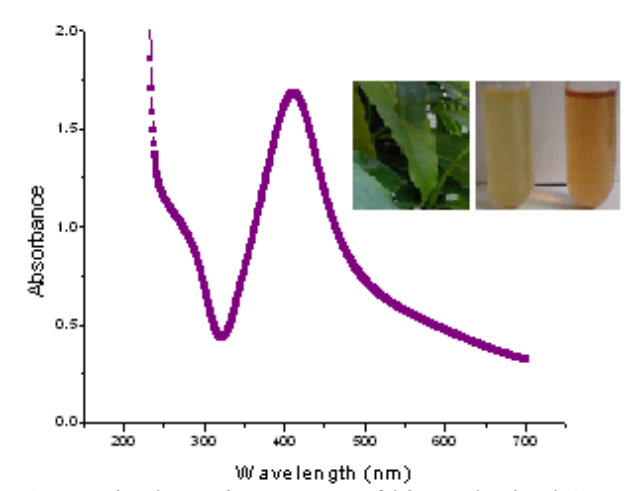

Fig. 1 UV-Vis absorption spectra of biosynthesized AgNPs. The inset: Saraca indica leaves and test tubes containing aqueous solutions of Saraca indica leaf extract (test tube on the left) and after reaction between extract and $\mathrm{AgNO}_{3}$ (test tube on the right).

\subsection{UV-Vis spectral analysis}

The formation of AgNPs by the reduction of the aqueous metal ions during exposure of the Saraca indica leaf extract can be easily evaluated by UV-Vis spectroscopy. The UV-Vis absorption spectrum was taken in the presence of leaf extract of Saraca indica (Fig. 1). The Surface plasmon resonance spectra of AgNPs obtained at $412 \mathrm{~nm}$ that showing no aggregation of nanoparticles in the UV-Vis spectrum.

\subsection{DLS analysis}

The synthesized AgNPs by Saraca indica leaf extract was analyzed by DLS to get the size distribution profile. The size distribution profile of AgNPs was found to be in the range of 21-62 nm (Fig. 2). The polydispersity index (PDI) of AgNPs was observed to be 0.292 which points out these particles are polydispersed.

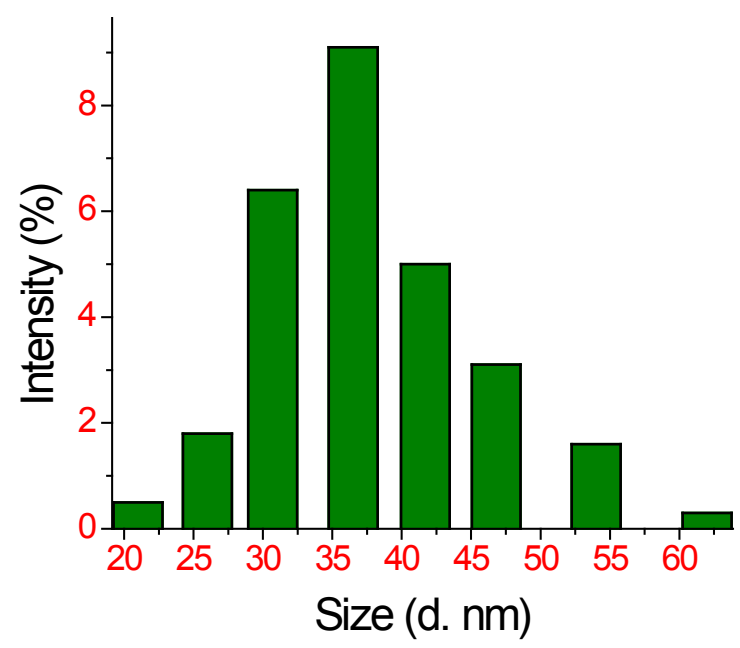

Fig. 2 DLS showing the size distribution profile of AgNPs

\subsection{TEM analysis}

The sample for TEM (Philips CM-10 TEM) characterization was prepared by putting a drop of suspension onto a copper grid and then allowed to dry at room temperature. TEM micrograph reveals that the AgNPs have spherical morphology with size range of 13-50 nm (Fig. 3). Micrograph also clearly depicted that there is no agglomeration.

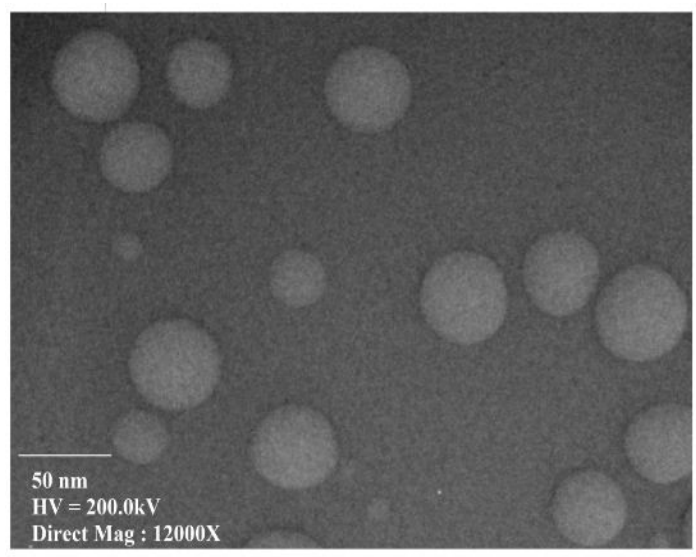

Fig. 3 TEM image of biogenic AgNPs synthesized by Saraca indica leaf extract.

Nano Biomed. Eng. 2013, 5(1),50-56 


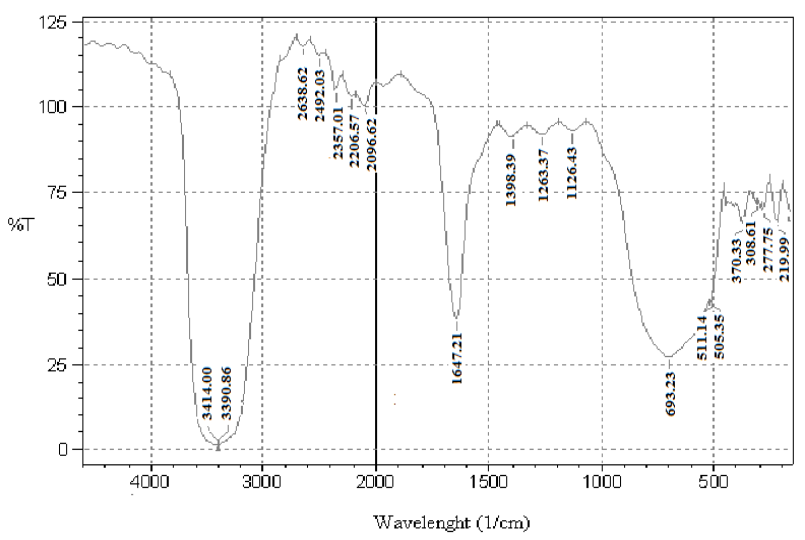

Fig. 4 FTIR spectra of biogenic AgNPs synthesized by reduction of Ag ions by Saraca indica leaf extract.

\subsection{FTIR analysis}

FTIR analysis is used to identify and to get an approximate idea of the possible biomolecules that are responsible for the reduction and stabilization of the AgNPs with the Saraca indica leaf extract (Fig. 4). In the range of 4000-3000 $\mathrm{cm}^{-1}$, spectrum shows a broad and strong intensity peak at nearly $3390 \mathrm{~cm}^{-1}$ and 3396 $\mathrm{cm}^{-1}$ which can be attributed to alcoholic or phenolic hydrogen bonded $\mathrm{O}-\mathrm{H}$ bonds. These peaks can also be due to the presence of amines $(\mathrm{N}-\mathrm{H})$ groups. In the range from $3000-2000 \mathrm{~cm}^{-1}$ spectrum gives five consecutive weak peaks which is due to alkynyl groups. A peak at $2638 \mathrm{~cm}^{-1}$ is due to the presence of carboxylic acid $\mathrm{O}-\mathrm{H}$ bonds. Spectrum gives a sharp and strong peak at 1647 $\mathrm{cm}^{-1}$ which could be due to the formation of hydrogen bonds between hydrogen atoms of aldehydes, carboxylic acids and alcohols at the surface. Hydrogen bonding tends to decrease the double-bond character of the $\mathrm{C}=\mathrm{O}$ group, which results in a shift of the $\mathrm{C}=\mathrm{O}$ absorption band to a lower frequency. The peak at $1647 \mathrm{~cm}^{-1}$ could also be attributed to $\mathrm{C}=\mathrm{C}$ stretching vibrations, about the amide $\mathrm{C}=\mathrm{O}$ and conjugated $\mathrm{C}=\mathrm{O}$ (ketones, aldehydes and esters) which proofs as a determinant for the presence of enzyme/proteins that are responsible for the reduction and stabilisation of AgNPs. In the region, from 1500$1000 \mathrm{~cm}^{-1}$ it gives consecutive three peaks with nearly same intensity which is due to alkanes, alcohol, ethers, carboxylic acid, esters, amines or nitro groups. A sharp peak at $698 \mathrm{~cm}^{-1}$ confirms about the alkenes $(\mathrm{C}-\mathrm{H})$ or phenol substitution bands $(\mathrm{C}-\mathrm{H})$. From the above analysis, this can be concluded that Saraca indica leaf extract contains enzymes/protein which might be responsible for reduction of the metal ions and reducing sugars like flavanones which are present on the surface of nanoparticles and provided stability.

\subsection{XRD analysis}

In this study, the XRD pattern obtained for AgNPs shows a characteristic high intensity peak at $2 \theta=79^{\circ}$ (Fig. 5). This peak corresponds to the pure silver showing Braggs reflections of (311) set of lattice plane, which is

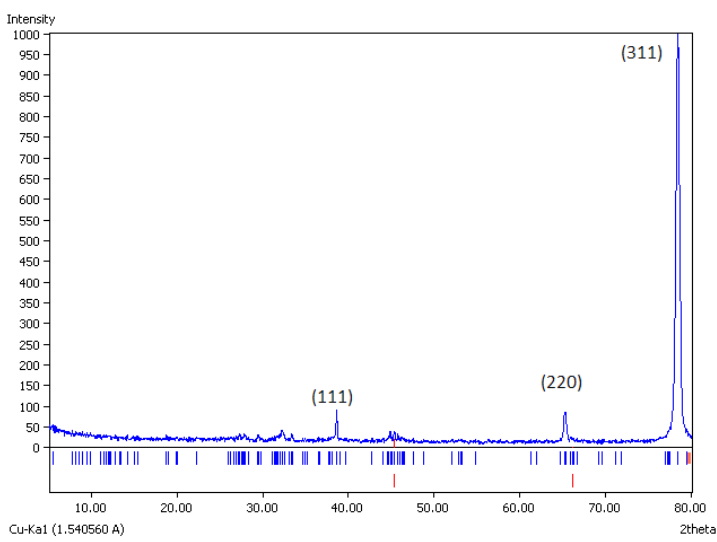

Fig. 5 XRD patterns of biogenic AgNPs synthesized by Saraca indica.

well coordinated with the existing Joint Committee on Powder Diffraction Standards (JCPDS File No. 04-0783). As estimated from the FWHM data of the peak, the size of silver nanocrystallites obtained was in accordance with the above characterizations. There are two more peaks but with a low intensity at around $38^{\circ}$ and $64.5^{\circ}$ corresponding to the (111) and (220) lattice planes (Fig. 5).

\subsection{Potential mechanism of biogenic synthesis of AgNPs}

Saraca indica is a medicinal plant from the ancient time. According to recent studies, some researchers have quoted that it has alkaloids, saponins, tannins, flavonoids and glycosides in its aqueous extract [28]. All these components are none other than glycosides except alkaloids. Out of them, tannins and flavonoids have the high antioxidative properties [29]. Tannin is a type of polyphenol and flavonoids are self-sufficient naturally

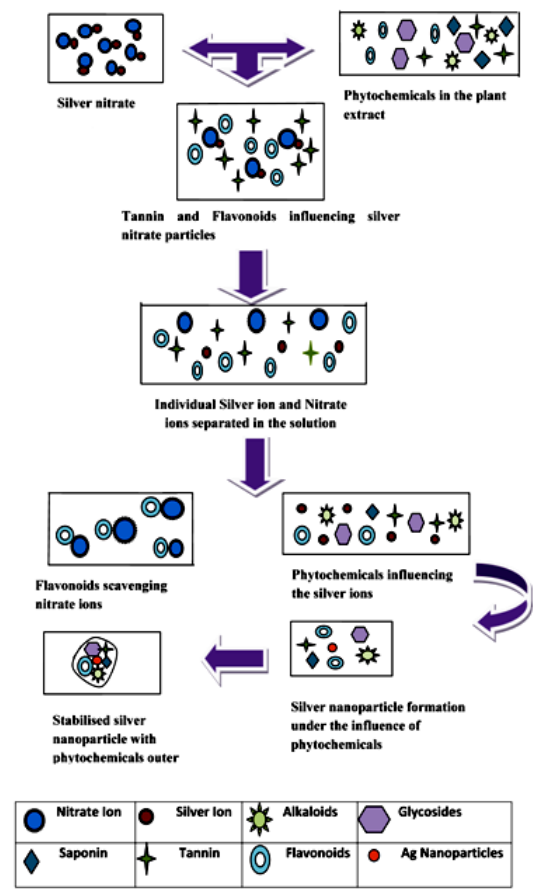

Fig. 6 Potential mechanism of synthesized biogenic AgNPs from Saraca indica leaf extract 
occurring antioxidants and iron chelators among the phytochemicals. From the recent studies on flavonoids, it has been concluded that, for good scavenging activity a catechol moiety on ring $\mathrm{B}$ is required. The 3-OH group in combination with a $\mathrm{C}_{2}-\mathrm{C}_{3}$ double bond increases the scavenging activity. The $3-\mathrm{OH}$ moiety can function as a chelation site and can also be oxidised [30].

Saponins have the foaming characteristics and consist of polycyclic aglycones attached to one or more sugar side chains. The aglycone part, which is also called sapogenin, is either steroid (C27) or a triterpene (C30). The foaming capability of saponins is caused by the combination of hydrophobic (fat-soluble) and hydrophilic (water-soluble) sugar part. Alkaloids have the medicinal value, but its reducing property has not reported yet.

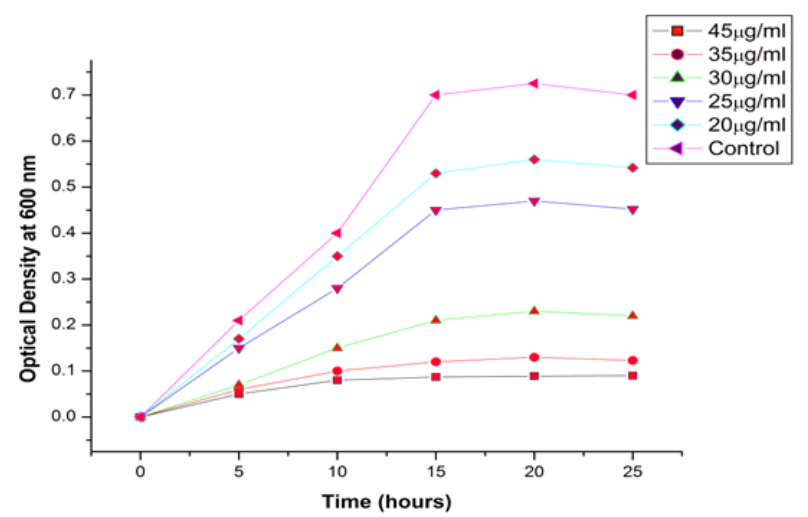

Fig. 7 Effect of various concentrations of biogenic AgNPs against E.coli DH5 $\alpha$ growth rate
Due to the above properties, the leaf extract molecules, act as strong antioxidants and will reduce the $\mathrm{Ag}^{+}$ions into Ag (Fig. 6). Flavonoids would scavenge all the free oxygen radicals present in the solution and thus contributes to the stability of nanoparticles. Hence all these phytochemicals are able to reduce silver ions and provide stability to the nanoparticles.

\subsection{Analysis of antibacterial activity of AgNPs}

The antibacterial activity of AgNPs was studied against E.coli $\mathrm{DH} 5 \alpha$ by measuring the optical density at $600 \mathrm{~nm}$ using UV-Vis spectrophotometer. The media were supplemented with $20-45 \mu \mathrm{g} / \mathrm{ml}$ concentration of AgNPs. On the basis of our experimental statistics, we can conclude that on increasing the concentration of AgNPs from 20-45 $\mu \mathrm{g} / \mathrm{ml}$, there is a decrease in optical density that shows the reduced bacterial growth of $E$. coli DH5 $\alpha$ (Fig. 7). It shows that these nanoparticles are susceptible for antibacterial action against the reported strain. The antibacterial activity of biogenic AgNPs was also analyzed by well diffusion method. The media were supplemented with various concentrations of biogenic AgNPs ranges from 20-45 $\mu \mathrm{g} / \mathrm{ml}$. After $25 \mathrm{~h}$ incubation, zone of inhibition was observed with different diameters. Inhibition zone varied with concentration of biogenic AgNPs, which is proved by our experiment (Fig. 8). Inhibition zone was not found in case of control culture because it does not have any exposure of AgNPs. These studies reveal the antibacterial nature of AgNPs.

Minimum inhibitory concentration (MIC) is the lowest concentration of an antibacterial agent that will inhibit the visible growth of a microorganism. MIC confirms
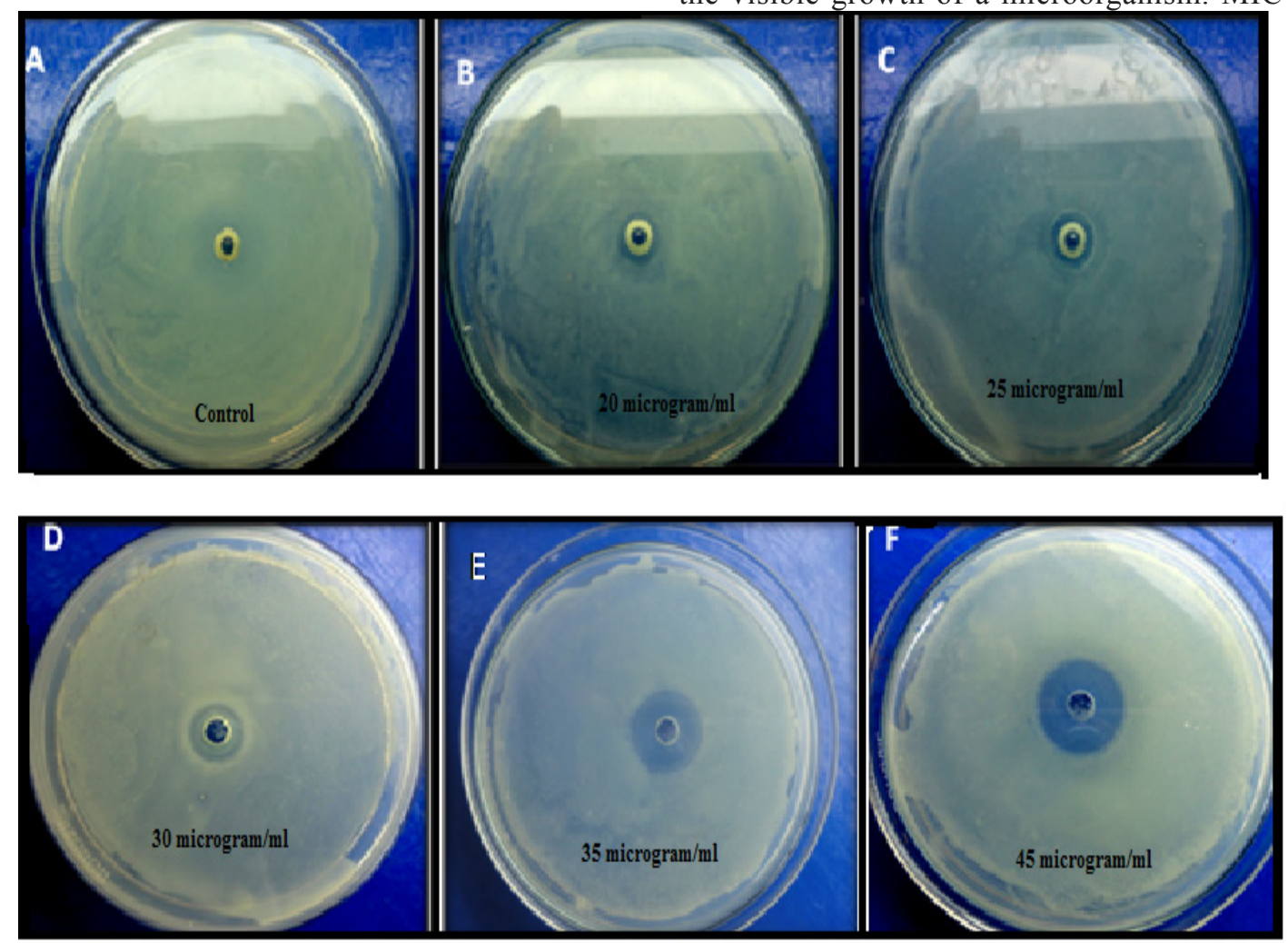

Fig. 8 Inhibition zone around the well impregnated with various concentration of biogenic AgNPs against E.coli DH5 $\alpha$. 
the resistance of the microorganism to an antibacterial agent and helps in monitoring the activity of the new microbial agents. A lesser MIC is a sign of an improved antibacterial agent. MIC determination is done by elaborative experiments on E.coli DH5 $\alpha$ cultures with different concentration of AgNPs, ranging from 20-45 $\mu \mathrm{g} /$ $\mathrm{ml}$. It was observed that $45 \mu \mathrm{g} / \mathrm{ml}$ concentration of AgNPs acts aseffective bactericides. Virtually there was no bacterial growth as optical absorption was insignificant. The growth curve indicates that minimum inhibitory concentration (MIC) is $20 \mu \mathrm{g} / \mathrm{ml}$ (Fig. 7). AgNPs shield the whole membrane of bacteria and their enzyme receptors, this leads to block the bio signals for vital activity. Finally the nanoparticles were accumulated in the cytoplasm and inhibit the metabolic process. This property of AgNPs makes it an effective bactericidal agent.

\section{Conclusion}

In the present study, we have synthesized AgNPs using Saraca indica leaf extract and evaluated their antibacterial activity. We are mainly paying attention at mechanistic approach to understand the biosynthesis of silver nanoparticles and their antibacterial activity. The leaf extract acts as reducing and capping agent (tannin and flavonoids are strong antioxidants, which will help in reduction of $\mathrm{Ag}^{+}$into $\mathrm{Ag}$ ). The size of AgNPs was obtained in the range of 13-50 nm. The FTIR analysis of the synthesized AgNPs also supports the proposed reaction mechanism. The particle shows effective antibacterial activity and hence can be used as an antibacterial agent with eco-friendly properties because the reaction is simple and feasible without any complications. The particles are spherical in shape as inferred from TEM images. The great tendency of the nanoparticles towards the toxicity for bacteria made them a good option for bio-medicinal application.

\section{Acknowledgements}

We would like to thank Dr. Ashok K. Chauhan, Amity Institute of Nanotechnology, Amity University (Noida, India) for encouragement and providing excellent facilities for the above work. Authors are also thankful to Dr. A. M. Jana, Professor and Head, Department of Biotechnology, College of Life Sciences, Gwalior (M.P) for giving his precious advice continuously. RMT thanks to Mrs. Nehul Saxena, ICGEB, New Delhi, India for help rendered in carrying out the experiments.

\section{References}

1 Saxena A., Tripathi R.M., Zafar F., Singh P., Green synthesis of silver nanoparticles using aqueous solution of Ficus benghalensis leaf extract and characterization of their antibacterial activity. Mater Lett. 2012; 67: 91-94.

2 Kumar V., Yadav C.S., Yadav K.S., Syzygium cumini leaf and seed extract mediated biosynthesis of silver nanoparticles and their characterisation. J. Chem. Sci. 2010; 85: 1301-1309.

3 Gittins D.I., Bethell D., Schiffrin D.J., Nichols R.J., A nanometre scale electronic switch consisting of a metal cluster and redoxaddressable groups. Nature 2000; 408: 67-69.

4 Mandal D., Bolander M.E., Mukhopadhyay D., Sarkar G., Mukherjee P., The use of microorganisms for the formation of metal nanoparticle and their applications. Appl. Microbiol. 2006; 69: 485-492.
5 Raveendran P., Fu J., and Wallen S L. Completely "green" synthesis and stabilization of metals nanoparticles. J. Am. Chem. Soc. 2003; 125: 13940-13941.

6 Ji M., Chen X., Wai C.M., Fulton J.L., Synthesizing and dispersing silver nanoparticles in a water-in-supercritical carbon dioxide microemulsion. J. Am. Chem. Soc. 1999; 121: 2631-2632.

7 Klaus T., Joerger R., Olsson E., Granquist C.G., Silver- based crystalline nanoparticles, microbially fabricated. Proc. Natt. Acad. Sci. USA. 1999; 96: 13611-13614.

8 Nair B., Pradeep T., Coalescence of nanoclusters and formation of submicron crystallites assisted by Lactobacillus strains. Cryst. Growth. Des. 2002; 2: 293-298.

9 Panacek A., Kvitek L., Prucek R., Kolar M., Veerova R., Pizurova N., Sharma V.K., Nevena T., Zboril R., Silver colloid nanoparticles: synthesis, characterisation and their antibacterial activity. J. Phys. Chem. B. 2007; 110: 16248-16253.

10 Jung W.K., Koo H.C., Kim K.W., Shin S., Kim S.H., Park Y.H., Antibacterial activity and mechanism of action of the silver ions in Staphylococcus aureus and E. coli. Appl. Environ. Microb. 2008; 74: 2171-2178.

11 Lee H.Y., Park H.K., Lee Y.M., Kim K., Park S.B., A practical procedure for producing silver nano film coated fabric and its antibacterial evaluation for biomedical applications. Chem. Commun. 2007; 28: 2959-2967.

12 Kumar V., Yadav S.K., Plant-mediated synthesis of silver and gold nanoparticle and their applications. J. Chem. Technol. Biot. 2009; 84: 151-157.

13 Mohanpuria P, Rana N.K., Yadav S.K., Biosynthesis of nanoparticles: technological concepts and future applications. J. Nanopart. Res. 2008; 10: 507-517.

14 Mukherjee P., Roy M., Mandal B.P., Dey G., Mukherjee K., Ghatak P.K., Tyagi J., Kale A.K.S.P., Green synthesis of highly stabilised nano crystalline silver particles by a non-pathogenic and agriculturally important fungus T asperellum. Nanotechnology. 2008; 19: 788-800.

15 Song J.K., Kim B.S., Rapid biological synthesis of silver nanoparticle using plant leaf extracts. Bioproc. Biosyst. Eng. 2009; 32: 79-82.

16 Shankar S.S., Ahmad A., Sastry M., Geranium leaf assisted biosynthesis of silver nanoparticles. Biotechnol. Progr. 2003; 19: 1627-1631.

17 Li S., Shen Y., Xie A., Yu X., Qui L., Zhang L., Zhang Q., Green synthesis of silver nanoparticles using Capsicum annuum extract. Green. Chem. 2007; 9: 852-858.

18 Shankar S.S., Rai A., Ahmad A., Sastry M., Rapid synthesis of Au, $\mathrm{Ag}$ and bimetallic Au core- Ag shell of nanoparticles using Neem (Azadirachta indica) leaf broth. Interface Sci. 2004; 275: 496-502.

19 Saxena Antariksh, Tripathi R.M., Singh R.P., Biological synthesis of silver nanoparticles by using onion (Allium cepa) extract and their antibacterial activity. Dig. J. Nanomater. Bios. 2010; 5: 427-432.

20 Nadagouda M.N., Varma R.S., Green synthesis of silver and palladium nanoparticles at room temperature using coffee and tea extract. Green Chem. 2008; 10: 859-862.

21 Gardea-Torresdey J.L., Gomez E., Peralta-Videa J.R., Parsons J.G., Troiani H., Jose-Yacaman M., Alfalfa sprouts of natural source for the synthesis of silver nanoparticles. Langmuir. 2003; 19: 1357-1361.

22 Haverkamp R.G., Marshall A.T., The mechanism of metal nanoparticle formation in plants: limits on accumulation. J. Nanopart. Res. 2009; 11: 1453-1463.

23 Huang J., Li Q., Sun D., Lu Y., Su Y., Yang X., Wang H., Wang Y., Shao W., Hong N.J., Chen C., Biosynthesis of silver and gold nanoparticles by novel Sundried Cinnamomum camphora Leaf," Nanotechnology. 2007; 18: 105104-105115.

24 Wei H., Chen C., Han B., Wang E., Enzyme colorimetric assay using unmodified silver nanoparticles. Anal. Chem. 2008; 80: 7051-7055.

25 Jain P., Pradeep T., Potential of silver nanoparticle-coated polyurethane foam as an antibacterial water filter. Biotechnol. Bioeng. 2005; 90: 59-63.

26 Shiraishi Y., Toshim N., Oxidation of ethylene catalysed by colloidal dispersions of poly (Sodium acrylate)-protected silver nanoclusters. Colloid Surface A. 2000; 169: 59-66.

27 Elechiguerra J.L., Burt J.L., Morones J.R., Camacho-Bragado A., Gao X., Lara H.H., Interaction of nanoparticles with HIV-1. J. Nanobiotechnol. 2005; 3: 6. 
30 Van Acker S.A., Van den berg D.J., Griffioen D.H., Van Bennekom W.P., Van der Vijgh W.J.F., Tormp M.N., Structural aspects of antioxidant activity of Flavonoids. Free Radical Bio. Med. 1996; 20: 331-342.

Copyright:(c) 2013 R.M. Tripathi et al. This is an open-access article distributed under the terms of the Creative Commons Attribution License, which permits unrestricted use, distribution, and reproduction in any medium, provided the original author and source are credited. 